\title{
Leiharbeit: Funktionswandel einer flexiblen Beschäftigungsform
}

Leiharbeit boomt. Doch trotz starker Expansion betrifft sie bislang nur einen Randbereich des Arbeitsmarktes. Bedeutsamer erscheint, dass sie die Einsatzbedingungen von Arbeit verändert. Sie lässt zunehmend flexible Rand- oder Parallelbelegschaften entstehen, die Stammbelegschaften teilweise ersetzen. Trotzdem werden Leiharbeitnehmer in der Mehrheit der Fälle in den Arbeitsbedingungen, vor allem beim Lohn und beim Zugang zu betrieblicher Weiterbildung, den Stammbeschäftigten nicht gleichgestellt.

\section{Problemstellung}

Leiharbeit expandiert dynamischer als die Gesamtbeschäftigung. Zwischen Mitte 2002 und 2007 hat sich die Zahl der Leiharbeitnehmer mehr als verdoppelt und liegt nun bei 731.000. Diese Entwicklung setzte bereits deutlich vor Beginn des letzten Konjunktur- und Beschäftigungsaufschwungs zeitgleich mit den Deregulierungen im Zuge der Hartz-Gesetze ein. Die Reform der Leiharbeit ermöglichte eine unbegrenzte Überlassungsdauer, hob das Wiedereinstellungsverbot auf und beseitigte das Synchronisationsverbot, das untersagte, den Arbeitsvertrag auf die Dauer des ersten Einsatzes zu befristen. Im Gegenzug führte sie das Prinzip der gleichen Bezahlung ein, von dem Tarifverträge allerdings abweichen können. Diese neuen rechtlichen Vorzeichen begünstigen veränderte Einsatzformen von Leiharbeit und erhöhen ihre Attraktivität für Entleiher. Diente sie ursprünglich primär dazu, situativ auftretenden Personalbedarf abzudecken, so mehren sich die Anzeichen, dass Betriebe sie vermehrt als flexible Rand- oder Parallelbelegschaften einsetzen und außerdem stärker zur Rekrutierung und Erprobung von Arbeitskräften nutzen. Ohne diesen qualitativen Wandel, so unsere zentrale These, lässt sich der quantitative Bedeutungszuwachs dieser Beschäftigungsform kaum erklären.

Wenngleich Leiharbeit mit gut $2 \%$ immer noch einen nur geringen Anteil der Gesamtbeschäftigung ausmacht, fällt die Dynamik ihrer Entwicklung auf. Dieses Phänomen steht im Zentrum des vorliegenden Beitrages. Er untersucht, welche Faktoren die Nutzung von Leiharbeit be- einflussen und liefert Hinweise zur Erklärung der starken Bedeutungszunahme der Leiharbeit.

Der Beitrag diskutiert zunächst, welche Rolle Leiharbeit im Spektrum betrieblicher Anpassungsstrategien spielt und zeigt die spezifischen Funktionen dieser Beschäftigungsform auf (Abschnitt 2). Anschließend legen wir den analytischen Rahmen für die nachfolgende empirische Untersuchung dar, erläutern die Datenbasis und beschreiben die Methode, bevor die Ergebnisse der bivariaten und der multivariaten Analyse ${ }^{1}$ vorgestellt werden (Abschnitt 3). Ein kurzes Fazit rundet den Beitrag ab (Abschnitt 4).

\section{Leiharbeit als personal- politisches Instrument}

\subsection{FLEXIBILISIERUNGSALTERNATIVE}

Personalstrategisch stellt Leiharbeit vor allem ein Flexibilisierungsinstrument dar. Sie gehört zu den zahlreichen Ansätzen, mit denen Betriebe den quantitativen Einsatz von Arbeit variieren können. Die optimale betriebliche Anpassungsstrategie ergibt sich aus einem Kostenkalkül, in das die Anpassungserfordernisse (saisonale, konjunkturelle Schwankungen, Urlaubs- oder Krankheitsvertretungen usw.) und die Anpassungsbedingungen (Qualifikationsstruktur, Verfügbarkeit der Arbeitskräfte, Rekrutierungs- und Entlassungskosten usw.) eingehen (Delsen 1996; Nienhüser/ Matiaske 2003). Die Palette der zur Verfügung stehenden Anpassungsvarianten lässt sich üblicherweise nach Formen externund intern-numerischer Flexibilität unterscheiden, die sich in weitere Subvarianten (funktional, monetär, temporal) auffächern (Atkinson 1984; Keller/Seifert 2007).

$\mathrm{Zu}$ den Formen extern-numerischer Flexibilität gehört der Einsatz von Randbelegschaften (Leiharbeitnehmer, befristet Beschäftigte, Aushilfen, freie Mitarbeiter mit Dienst- oder Werkverträgen, usw.). Im Vergleich zu den Stammbelegschaften sind sie aufgrund ihrer geringen oder allseits vorhandenen Qualifikationen und Fertigkeiten leichter ersetzbar, für das effiziente Funktionieren der Betriebe weniger bedeutsam, verursachen außerdem geringere Entlassungs- sowie Wiederbeschäftigungskosten und tragen deshalb ein höheres Beschäftigungsrisiko (Cappelli/Neumark 2004). Bei Nachfrageschwankungen variieren die Betriebe den Arbeitseinsatz so weit wie möglich durch interne Flexibilität sowie durch die Anzahl der Beschäftigten in den Randbelegschaften. Flexibilität wird auch den Stammbelegschaften abverlangt. Als Gegenleistung für vergleichsweise höhere Beschäftigungssicherheit haben sie interne Flexibilität in Form numerischer (Arbeitszeit), monetärer und funktionaler Anpassung zu leisten (Bellmann et al. 1996;

\footnotetext{
1 Unser Dank gilt Martin Behrens und Ivar Krumpal, die uns bei der Erstellung und Schätzung des Modells mit konstruktiver Kritik und Rat zur Seite standen.
}

Hartmut Seifert, Dr., Leiter der Abteilung WSI in der Hans-Böckler-Stiftung. Arbeitsschwerpunkte: Arbeitszeitpolitik und Arbeitsmarktforschung. e-mail: hartmut-seifert@boeckler.de Wolfram Brehmer, Wissenschaftler im WSI in der Hans-Böckler-Stiftung. Arbeitsschwerpunkte: Betriebs- und Personalräteforschung. e-mail: Wolfram-Brehmer@boeckler.de 
Kalleberg 2001; Seifert/Massa-Wirth 2005; Massa-Wirth 2007).

Die Formen interner und externer Flexibilität können sich ersetzen, aber auch ergänzen. So stellt beispielsweise Leiharbeit eine Alternative zu befristeter Beschäftigung oder zur Variation der Arbeitszeit von Stammbeschäftigten dar. In der betrieblichen Praxis dominieren komplexe Flexibilisierungspakete. Betriebe nutzen neben Leiharbeit gleichzeitig auch Überstunden, Arbeitszeitkonten und Befristungen, also verschiedene Formen sowohl externer als auch interner Flexibilität (Promberger 2005).

Leiharbeit ist durch Besonderheiten charakterisiert, die sie sowohl unter Flexibilitäts- als auch Kostenaspekten von anderen Formen externer Flexibilität abhebt und für Arbeitgeber attraktiv macht. Unter Flexibilitätsaspekten liegen die Stärken von Leiharbeit darin, dass Arbeitskräfte in den nachgefragten Qualifikationen ad hoc ohne lange Suchzeiten verfügbar sind, deren Einsatz sich genau mit den benötigten Zeiten des Arbeitskräftebedarfs synchronisieren lässt. Die Kostenaspekte haben mit der besonderen arbeitsrechtlichen Konstruktion der Leiharbeit zu tun. Bei dieser Beschäftigungsform fallen im Unterschied zu Normalarbeitsverhältnissen oder befristeter Beschäftigung das Arbeits- und das Beschäftigungsverhältnis auseinander (Seifert 1988). Leiharbeitnehmer schließen ihren Arbeitsvertrag mit dem Verleiher ab, mit dem sie auch Lohn und Arbeitszeit vereinbaren. Die Arbeitgeberrolle bleibt beim Verleiher. Die zu erbringende Arbeitsleistung definiert jedoch der Entleiher, der das Weisungsrecht und damit partiell Arbeitgeberfunktionen ausübt (Urban-Crell/ Schulz 2003).

Wegen dieses besonderen Dreiecksverhältnisses zwischen Verleiher, Leiharbeitnehmer und Entleiher können die Arbeitsbedingungen, hauptsächlich Lohn und Arbeitszeit, vom Niveau vergleichbarer Stammkräfte nach unten abweichen. Wie empirische Analysen zeigen (z. B. Jahn/ Rudolph 2002; Nienhüser/Matiaske 2003; Brehmer/Seifert 2007), erhalten Leiharbeitnehmer deutlich geringere Löhne als vergleichbare Beschäftigte im Normalarbeitsverhältnis. Bis in die Mitte der 1990er Jahre hatte sich der Lohnabstand zwischen Zeitarbeit und regulärer Arbeit noch ausgeweitet. Er lag für gering qualifizierte Arbeit deutlich höher als für höher Qualifizierte $^{2}$ (Kvasnicka/Werwatz 2003). Dem
Entleiher entstehen jedoch höhere Arbeitskosten, da er an den Verleiher einen über dem Lohn des Leiharbeitnehmers liegenden Verrechnungssatz zu entrichten hat. Dessen Höhe variiert mit der Spezifität der Tätigkeiten und dem Qualifikationsgrad der Beschäftigten. Schätzungen gehen davon aus, dass die Verrechnungssätze bei standardisierten Tätigkeiten, dem überwiegenden Einsatzbereich von Leiharbeit, aber immer noch deutlich (bis zu $25 \%$ ) unter den Kosten von Festanstellungen liegen (Miegel et al. 2007). Mit steigendem Qualifikationsgrad der Arbeitskräfte wächst die Wahrscheinlichkeit, dass die Verleihsätze die vergleichbaren Arbeitskosten bei Festanstellungen übertreffen (Ammermüller et al. 2003).

Diese Kostenvergleiche berücksichtigen nur einen Teil der gesamten Arbeitskosten. Es gehen nur die unmittelbaren Arbeitskosten ein, die lediglich die Bruttolohn- und Gehaltskosten einschließlich der Sozialbeiträge des Arbeitgebers erfassen. Unberücksichtigt bleibt, dass bei Leiharbeit gegenüber anderen Formen externer Flexibilität dem Entleiher keine Rekrutierungsund Entlassungskosten entstehen, keine eigene Personalreserve vorzuhalten ist, weder durch Senioritäts- oder Freistellungsregelungen noch durch Krankheit verursachte Kosten anfallen und der Arbeitskräftebedarf ad hoc ohne lange Suchzeiten in der erforderlichen Quantität und Qualität zur Verfügung steht (Promberger 2006).

\subsection{FUNKTIONEN}

Unter den beschriebenen Kosten- und Flexibilisierungsaspekten kann Leiharbeit je nach Problemkonstellation anderen Anpassungsformen überlegen sein und vor allem drei personalstrategische Funktionen erfüllen. Traditionell nutzen Betriebe diese Beschäftigungsform vorrangig, um Stabilität im Personaleinsatz zu sichern (Håkansson/Isidorsson 2008). Dies ist der Fall, wenn situativ Stammarbeitskräfte infolge von Krankheit, Urlaub oder Freistellungszeiten ausfallen und befristet $\mathrm{zu}$ ersetzen sind. Lange Zeit dominierte diese Funktion (Promberger 2005).

Leiharbeit kann zweitens der betrieblichen Personalrekrutierung dienen. Betriebe können Leiharbeitskräfte unverbindlich erproben, ohne Kündigungsschutzregelungen zu beachten. Zudem kann die Einschaltung von Verleihfirmen bei der Rekrutierung kostengünstiger sein, da diese nicht nur über Routinen in der Auswahl geeigneter Bewerber verfügen und das Risiko von Fehlbesetzungen verringern, sondern diesen Prozess auch kostengünstiger organisieren können (economies of scale) (Houseman 2001, S. 157).

Leiharbeit kann drittens Stammarbeitskräfte temporär ersetzen oder den Bedarf an zusätzlichen Arbeitskräften ganz oder teilweise abdecken. In diesem Fall übernimmt diese Beschäftigungsform die Funktion einer flexiblen Rand- oder Parallelbelegschaft. Als saisonaler oder gar konjunktureller Puffer ersetzt oder ergänzt sie zeitweise Teile der Kernbelegschaften. Starke saisonale und konjunkturelle Einflüsse prägen das Verlaufsmuster der Leiharbeit (Brenke/Eichhorst 2008). Mit wachsendem Gewicht dieser Funktion verändern sich die Einsatzformen von Leiharbeit. Die Einsatzzeiten werden länger, die Zahl der in einem Entleihbetrieb beschäftigten Leiharbeitnehmer steigt (Intensivnutzer). Ihr Einsatz gilt nicht mehr nur der Vertretung oder Unterstützung einzelner Beschäftigter, um Arbeitsspitzen zu bewältigen, sondern einzelne Funktionsbereiche werden zu weiten Teilen von Leiharbeitnehmern übernommen (Promberger 2006; Klemm et al. 2008).

Ablesen lassen sich die veränderten $\mathrm{Ge}$ wichtungen in den personalstrategischen Funktionen ferner daran, dass Verleiher ihre Arbeitgeberrolle aktivieren, ihre personalpolitischen Funktionen im Rahmen von „On-site-Management“ ausweiten und vor Ort beim Entleiher ausüben (Promberger 2006; Miegel et al. 2007; Klemm et al. 2008). Diese Personalleistung setzt in aller Regel einen nicht nur kurzfristigen Mindestbedarf an Leiharbeit voraus (Böhm 2005). Der Kunde spezifiziert seinen Personalbedarf in quantitativer und qualitativer Hinsicht. Der Verleiher übernimmt die profilgenaue Rekrutierung des Personals ebenso wie dessen Betreuung und sorgt bei Ausfällen für raschen Ersatz. Ein professionalisiertes externes Personalmanagement aus einer Hand hilft, Fehlbesetzungen zu verringern und Einarbeitungszeiten zu verkürzen. Der rasche Auf- und ebenso rasche, zudem meist konfliktarme, auf jeden Fall aber kostenlose Abbau von Personal gestat-

2 Diese Befunde beziehen sich auf eine Situation, bevor für den weit überwiegenden Bereich der Leiharbeit Tarifverträge abgeschlossen wurden, die eine untere Lohngrenze einführten. 
tet, einen Teil der Belegschaften maßgeschneidert an Nachfrageschwankungen anzupassen.

In welchem Verhältnis die personalstrategische Entscheidung für Leiharbeit oder andere Formen der Flexibilität fällt, hat jeder Betrieb vor dem Hintergrund seiner spezifischen Kostenstrukturen, Arbeitsorganisation und alternativen Handlungsmöglichkeiten herauszufinden. An Grenzen stößt Leiharbeit bei Tätigkeiten, bei denen komplexeres betriebliches Erfahrungswissen und eingespielte Teamarbeit abverlangt werden, ein Vertrauensschutz für betriebliche Wissensbestände erforderlich ist oder enge Kundenbeziehungen bestehen (Promberger 2007). Um den potenziellen Einsatzbereich für Leiharbeit auszuweiten, beginnen Betriebe, bereits bei Planung und Gestaltung der Arbeitsorganisation diese personalpolitische Alternative in das Entscheidungskalkül einzubeziehen. Je einfacher die Arbeitsabläufe sind, desto kürzer können die Einarbeitungsund Anlernzeiten und desto größer wird der potenzielle Einsatzbereich für Leiharbeit sein (Håkansson/Isidorsson 2008).

\section{Empirische Analyse}

\subsection{ANALYTISCHER RAHMEN}

Die Expansion der Leiharbeit hat mehrere Gründe. Auslöser ist offensichtlich die Deregulierung des rechtlichen Rahmens im Zuge der Hartz-Gesetze. Der Aufschwung dieser Beschäftigungsform fällt mit dem Zeitpunkt ihrer gesetzlichen Änderungen zusammen, die mit dem ersten Hartz-Gesetz zum Jahresbeginn 2003 in Kraft traten, allerdings eine einjährige Übergangsfrist vorsahen. Der Beginn des Konjunkturaufschwungs ist deutlich später für den Jahreswechsel 2004/05 zu datieren (IMK 2007); noch später, im ersten Quartal 2006, setzte der Beschäftigungsaufschwung ein, also fast drei Jahre, nachdem die Expansion der Leiharbeit begann. Natürlich hat auch die konjunkturelle Erholung für einen Schub bei der Leiharbeit gesorgt. Drittens dürften veränderte Rahmenbedingungen auf der Angebotsseite des Arbeitsmarktes eine Rolle spielen. Die im Zuge der HartzReformen verschärften Bedingungen zur Arbeitsaufnahme (Zumutbarkeitsregelungen) verstärken den Druck auf die Konzes- sionsbereitschaft von Arbeitslosen, im Vergleich zu früheren Tätigkeiten wesentlich geringer entlohnte Beschäftigungsverhältnisse anzunehmen. Die weiteren Erörterungen richten sich allein auf die Nachfrageseite und die sie beeinflussenden Faktoren.

Unter den veränderten rechtlichen Rahmenbedingungen hängt die Nachfrage nach Leiharbeit davon ab, inwieweit die Betriebe die verschiedenen Funktionen dieser Beschäftigungsform nutzen. Denkbar ist, dass Betriebe Leiharbeit gleichzeitig in unterschiedlichen Bereichen sowohl zum Einsatz von ad hoc-Aushilfen als auch zur Erprobung sowie zur Ausweitung oder Substitution von Stammbeschäftigten einsetzen. Die drei Funktionen können sich überlagern und das Ausmaß der Leiharbeit steigern. Insofern lässt sich als Hypothese formulieren, dass die Höhe des Leiharbeitereinsatzes davon abhängt, in welchem Maße die Betriebe die verschiedenen Funktionen miteinander kombinieren und ergänzend nutzen. Hohe Leiharbeitsanteile sind vor allem dort zu erwarten, wo sowohl die Screening- als auch die flexible Randbelegschaftsfunktion eine bedeutsame Rolle in der betrieblichen Personalstrategie spielen. Empirisch lassen sich diese Funktionen mithilfe des hier verwendeten Datenmaterials nur indirekt abbilden. Zudem erfüllen sie nicht immer das Kriterium der Trennschärfe, sondern überlappen sich teilweise. Es wird angenommen, dass folgende Indikatoren Aussagen über die Funktionen von Leiharbeit zulassen:

(1) Von der Screening-Funktion ist auszugehen, wenn Entleiher zumindest einen Teil der Leiharbeitnehmer übernehmen. Je höher der Anteil der übernommenen Leiharbeitnehmer, desto größer dürfte die Bedeutung der Erprobungsfunktion sein.

(2) Die Rolle der flexiblen Randbelegschaften dürfte stark von Kostengesichtspunkten bestimmt werden. Zu vermuten ist, dass hohe Anteile von Leiharbeit eher in Hochlohn- als in Niedriglohnbereichen anzutreffen sind. Aus Sicht der Verleihbetriebe lohnt der Verleih nur, wenn die Nutzerbetriebe bereit sind, deutlich über den Tariflöhnen der Leiharbeitnehmer liegende Verrechnungssätze zu zahlen. Je größer die Differenz zwischen dem Lohnniveau der Verleiher und dem der Entleiher ist, desto wahrscheinlicher ist der Einsatz dieser Beschäftigungsform. Und umgekehrt verliert Leiharbeit in dem Maße an Attraktivität, wie die Effektivlöhne in den Entleihbetrie- ben das Niveau in den Verleihbetrieben unterschreiten. Da der verwendete Datensatz keine Informationen über die betrieblichen Lohnniveaus liefert, dient das Branchenmerkmal als grober Anhaltspunkt. Niedriglohnsektoren wie der Handel erscheinen für den Einsatz von Leiharbeit unattraktiver als z. B. das Investitionsgütergewerbe mit deutlich höheren Löhnen. Dagegen dürfte der Aspekt der Lohndifferenz an Bedeutung verlieren, wenn Leiharbeit vorrangig dem Screening oder der Überbrückung von ad hoc-Personalbedarfen dient.

(3) $\mathrm{Zu}$ vermuten ist ferner, dass der Anteil der Frauenbeschäftigung die Funktion der flexiblen Randbelegschaft negativ beeinflusst. Für diese Annahme spricht, dass Frauen eher in Niedriglohnbereichen beschäftigt sind und außerdem zu einem wesentlich höheren Anteil als Männer Teilzeitarbeit leisten. Teilzeitbeschäftigung stellt aufgrund der vergleichsweise großen zeitlichen Variationsmöglichkeiten, die zudem rasch und vergleichsweise kostengünstig mithilfe eingearbeiteter Arbeitskräfte nutzbar sind, eine alternative Flexibilisierungsvariante dar und dürfte deshalb den Einsatz von Leiharbeit dämpfen.

(4) Leiharbeit kann befristete Beschäftigung ergänzen oder auch ersetzen. Die zweite Alternative dürfte vor allem bei wiederkehrendem Arbeitsbedarf eine Rolle spielen, da ein Arbeitnehmer sachgrundlos nicht ein zweites Mal bei demselben Arbeitgeber beschäftigt werden darf (Teilzeit- und Befristungsgesetz $\$ 14$ Abs. 2). Ein Indikator für wiederkehrenden Arbeitsbedarf wäre ein saisonal bedingter Einsatz. In dem Maße, wie Leiharbeit genutzt wird, um saisonale Schwankungen abzufedern, ist eine steigende Einsatzquote zu erwarten.

(5) Mit zunehmender Verleihdauer dürfte die Wahrscheinlichkeit steigen, dass es sich nicht nur um eine situative Überbrückung von Arbeitsengpässen oder Screening handelt, sondern Leiharbeit auch Stammkräfte ergänzt oder ersetzt. In dem Maße, wie sich Anzeichen finden, dass Leiharbeit Stammarbeitskräfte ersetzt, dürfte sich auch der quantitative Einsatz erhöhen. Leiharbeit übernimmt die Funktion von flexiblen Randbelegschaften. Der Einsatz bleibt nicht nur auf die Überbrückung einzelner temporärer Vakanzen beschränkt.

(6) Sofern Einsatzbetriebe über eigene Leiharbeitsfirmen verfügen, erscheint es wahrscheinlich, dass sie Leiharbeit intensiv nutzen und über diesen personalstrategischen 
Tabelle 1: Strukturmerkmale der Leiharbeit nach Wirtschaftsbereichen

\begin{tabular}{|c|c|c|c|c|c|c|c|c|c|}
\hline & $\begin{array}{l}\text { Ins- } \\
\text { gesamt }\end{array}$ & $\begin{array}{l}\text { Grundstoffe/ } \\
\text { Produktions- } \\
\text { güter }\end{array}$ & $\begin{array}{l}\text { Investitions-/ } \\
\text { Gebrauchs- } \\
\text { güter }\end{array}$ & $\begin{array}{l}\text { Verbrauchs- } \\
\text { güter }\end{array}$ & Bau & Handel & $\begin{array}{c}\text { Verkehr/ } \\
\text { Nachrichten }\end{array}$ & $\begin{array}{l}\text { Kredit/ } \\
\text { Versiche- } \\
\text { rungen }\end{array}$ & $\begin{array}{l}\text { Sonstige } \\
\text { Dienst- } \\
\text { leistungen }\end{array}$ \\
\hline Betriebe mit Leiharbeit (Anteil in \%) & 37,3 & 67,6 & 59,9 & 39,8 & 41,9 & 23,3 & 39,6 & 18,0 & 32,0 \\
\hline \multicolumn{10}{|l|}{ In der Gruppe mit Leiharbeit } \\
\hline Anteil Leiharbeiter (in \% im Mittel) & 8,2 & 9,4 & 9,2 & 6,8 & 19,4 & 5,8 & 8,1 & 2,6 & 5,9 \\
\hline Einsatzdauer (in Monaten im Mittel) & 7,3 & 8,2 & 8,4 & 4,7 & 6,9 & 6,2 & 5,3 & 8,4 & 7,6 \\
\hline \multicolumn{10}{|l|}{$\begin{array}{l}\text { Betriebe mit... } \\
\text { (Anteil in \%) }\end{array}$} \\
\hline ... wachsender Leiharbeit & 54,6 & 56,7 & 58,9 & 60,0 & 57,7 & 48,9 & 50,8 & 45,5 & 52,8 \\
\hline ... unveränderter Leiharbeit & 31,8 & 29,3 & 25,1 & 31,0 & 29,9 & 36,4 & 34,5 & 44,5 & 34,9 \\
\hline ... abnehmender Leiharbeit & 13,7 & 14,0 & 16,0 & 9,0 & 12,4 & 14,7 & 14,7 & 10,0 & 12,2 \\
\hline ... schlechterer Entlohnung bei Leiharbeit & 76,4 & 87,6 & 82,8 & 87,6 & 70,2 & 66,7 & 82,9 & 81,0 & 67,9 \\
\hline ... Substitution regulärer Beschäftigung & 25,9 & 25,1 & 17,7 & 25,7 & 25,3 & 27,3 & 24,7 & 33,4 & 31,9 \\
\hline ... Übernahme von Leiharbeitern & 64,1 & 76,7 & 73,4 & 57,7 & 58,9 & 59,6 & 67,1 & 41,8 & 58,1 \\
\hline ... eigener Verleihfirma & 7,1 & 5,6 & 3,4 & 5,2 & 2,9 & 6,7 & 6,4 & 9,3 & 12,9 \\
\hline
\end{tabular}

Weg Teile der Stammbelegschaft ergänzen oder ersetzen. Bei unternehmenseigenen Verleihfirmen kann es sich teilweise um Transfergesellschaften handeln, die ehemalige Stammarbeitskräfte beschäftigen, die aufgrund ihrer bisherigen beruflichen Erfahrungen mit den internen Arbeitsabläufen des ehemaligen Unternehmens vertraut und deshalb dort kurzfristig als produktive Reserve einsetzbar sind.

\subsection{DATEN}

Die folgende Analyse verwendet die Daten der WSI-Betriebsrätebefragung 2007 (vgl. zur Methode Schäfer in diesem Heft), deren Grundgesamtheit Betriebsstätten in Deutschland mit Betriebsrat ab 20 Beschäftigten bilden. Die Stichprobenziehung erfolgte über eine disproportional geschichtete Zufallsziehung aus der Betriebsstättendatei der Bundesagentur für Arbeit. Daher sind die hier vorgestellten Angaben mittels des Horvitz-Thompson-Quotienten-Schätzers gewichtet, um dem Ziehungsprozess Rechnung zu tragen. Ansprechpartner im Betrieb war der Betriebsrat. Die Befragung fand zwischen September und Dezember 2007 statt. Angaben zum aktuellen Leiharbeitseinsatz beziehen sich auf diesen Zeitraum. Angaben zur Veränderung des Leiharbeitnehmereinsatzes wurden retrospektiv für die letzten beiden Jahre erhoben. Unter den 2.070 befragten Betriebsräten bzw. Betrieben gaben 37,3\% an, aktuell Leiharbeitnehmer zur Belegschaft zu zählen. In der bivariaten Analyse werden je nach gewähltem Bezug entweder die Angaben aller 2.070 befragten Betriebe oder nur die Angaben der Betriebe mit Leiharbeit betrachtet. Die multivariate
Analyse beschränkt sich ausschließlich auf die Betriebe mit Leiharbeit. Einige Betriebe sind wegen fehlender Angaben ausgeschlossen. Insgesamt gehen 720 Betriebe in die multivariate Analyse ein.

\subsection{BIVARIATE ANALYSE}

Nach den Befunden der WSI-Betriebsrätebefragung nutzten Leiharbeit im Herbst 2007 gut $37 \%$ der Betriebe mit Betriebsrat und mindestens 20 Beschäftigten (Tabelle 1). Der Anteil der Nutzerbetriebe steigt mit der Betriebsgröße. Er streut ferner zwischen den Wirtschaftsbereichen. Den höchsten Anteilswert weist das Grundstoffund Produktionsgütergewerbe auf, den niedrigsten der Bereich Versicherungen und Kreditgewerbe. Eine vergleichsweise geringe Rolle spielt diese Beschäftigungsform auch im Handel. Diese Branchenunterschiede verweisen auf unterschiedliche Einsatzbedingungen. Versicherungen und Kreditgewerbe sowie Handel sind weniger konjunkturreagibel als Branchen im produzierenden Gewerbe. Beim Handel dürfte ferner das geringe Lohnniveau den Einsatz von Leiharbeit unattraktiv erscheinen lassen, während bei Banken und Versicherungen die erforderliche Vertrautheit mit Arbeitsabläufen und -besonderheiten (Vertrauen, Kundenbeziehungen) Grenzen setzt. Im Baugewerbe bleibt Leiharbeit auf das Ausbaugewerbe beschränkt. Im Bauhauptgewerbe, dem gemessen an der Beschäftigtenzahl kleineren Teilbereich des Baugewerbes, bleibt sie auch nach den Hartz-Reformen weiterhin verboten.

In den Nutzerbetrieben beträgt der durchschnittliche Anteil der Leiharbeiter an allen Beschäftigten gut $8 \%$. Als Inten- sivnutzer mit einem Leiharbeiteranteil von mehr als $20 \%$ der Beschäftigten (Promberger 2006) können gut $11 \%$ der Betriebe bezeichnet werden. ${ }^{3}$ Auffallend ist, dass im Baugewerbe der durchschnittliche Anteil der eingesetzten Leiharbeiter deutlich den Gesamtdurchschnitt übertrifft, obwohl der Anteil der Leiharbeit nutzenden Betriebe nur wenig über dem Durchschnitt liegt. In diesem Bereich ist auch der Anteil der Intensivnutzer besonders hoch. Das betrifft vor allem die unteren Betriebsgrößenklassen und ist insofern teilweise ein Größenklasseneffekt. Zu den Werten des Bausektors ist relativierend anzumerken, dass ein Teil der Betriebe dieses Sektors, nämlich diejenigen des Bauhauptgewerbes, keinen Gebrauch von dieser Beschäftigungsform machen kann, während ein anderer Teil dieses Bereiches, das Ausbaugewerbe, Leiharbeit offensichtlich umso stärker einsetzt.

In den letzten Jahren zwischen 2005 und 2007 hat nach den Aussagen der befragten Betriebsräte mehr als die Hälfte (55\%) der Nutzerbetriebe den Einsatz der Leiharbeit ausgeweitet, ein wesentlich kleinerer Teil (14\%) hat ihn reduziert. Die Expansion ist ohne große Unterschiede in Betrieben aller Größenklassen beobachtbar. Differenziert nach Wirtschaftsbereichen zeigt sich dagegen ein anderes Bild. Den relativ stärksten Anstieg verzeichnet das Verbrauchsgütergewerbe, wo $60 \%$ der Betriebsräte aus den Nutzerbetrieben einen Zuwachs angeben, gegenüber nur $46 \%$ im Kredit- und Versicherungsgewerbe. Die

3 Auf einen mit gut $10 \%$ nahezu identischen Wert
kommt eine Analyse auf Basis des IAB-Betriebspanels (Bellmann/Kühl 2007). 
durchschnittliche Einsatzdauer von Leiharbeitnehmern beziffern die Betriebsräte auf gut ein halbes Jahr. Dieser Wert streut nur wenig zwischen den Betriebsgrößenklassen, stärker dagegen zwischen den Wirtschaftsbereichen. Vergleichsweise kurz, mit 4,7 Monaten, sind die Einsatzzeiten im Verbrauchsgütergewerbe, mit 8,4 Monaten dagegen deutlich länger im Investitionsgüter- und Gebrauchsgütergewerbe sowie im Bereich Kredit und Versicherungen. Etwas über ein Drittel (37\%) der Betriebsräte aus Nutzerbetrieben berichtet über längere Einsatzzeiten.

Leiharbeitnehmer werden in der überwiegenden Mehrheit der Betriebe nach den Aussagen der Betriebsräte schlechter entlohnt als entsprechende Stammkräfte. Die Lohndifferenz beträgt durchschnittlich $29 \%$. Diese Befunde decken sich mit denen anderer Untersuchungen (Promberger 2006; Nienhüser/Matiaske 2003; Brehmer/ Seifert 2007). Lohndifferenzen bestehen aber nicht in allen Betrieben. In fast einem Viertel der Entleihbetriebe erhalten Leiharbeitnehmer den gleichen Lohn wie Stammkräfte. Benachteiligt sind Leiharbeitnehmer auch bei der beruflichen Weiterbildung; etwa $60 \%$ der Einsatzbetriebe beziehen sie nicht in dem Maße wie vergleichbare Stammkräfte in Trainingsaktivitäten ein.

Fast zwei Drittel der befragten Nutzerbetriebe haben in den letzten zwei Jahren Leiharbeit als Rekrutierungsquelle genutzt und Leiharbeitnehmer übernommen. Ein gutes Viertel der Betriebe hat Leiharbeit aber auch eingesetzt, um reguläre Stellen zu ersetzen. Substitutionseffekte lassen sich im Bereich des Kredit- und Versicherungsgewerbes häufiger beobachten als im Investitionsgüter- und Gebrauchsgütergewerbe. Insgesamt fällt die Substitutionsquote um etwa die Hälfte kleiner aus als die Übernahmequote. Diese Befunde lassen sich als Hinweis interpretieren, dass Leiharbeit in betrieblichen Personalstrategien sowohl als Screeninginstrument als auch in der Funktion der flexiblen Randbelegschaft eine bedeutsame Rolle spielt, wobei insgesamt gesehen der erstgenannten Rolle offensichtlich das größere Gewicht zufällt.

\subsection{MULTIVARIATE ANALYSE}

\section{METHODE}

Wir untersuchen nachfolgend den Zusammenhang zwischen den Leiharbeitnehmer- anteilen an den Gesamtbeschäftigten und einer Reihe erklärender Variablen, welche wir aus oben ausgeführten Überlegungen $\mathrm{zu}$ den Leiharbeitsfunktionen herleiten (Abschnitt 3.1). Zur Schätzung der Leiharbeitnehmeranteile verwenden wir eine multiple lineare Regression nach der Methode der kleinsten Quadrate (OLS). Da wir u. a. Strukturmerkmale der Leiharbeit als erklärende Variablen in das Modell einführen, beschränken wir die Analyse auf die Betriebe mit Leiharbeit. Die abhängige Variable „Leiharbeitnehmeranteil“ kann somit theoretisch Werte größer Null bis einschließlich 100 annehmen. Tatsächlich werden jedoch wenige Leiharbeitnehmeranteile über $50 \%$ beobachtet; der Leiharbeitnehmeranteil streut rechtsschief. Deshalb verwenden wir zur Schätzung der Standardfehler ein robustes Schätzverfahren, welches die Homoskedastizität der Residuen nicht voraussetzt. ${ }^{4}$

An erklärenden Variablen führen wir neben betrieblichen Angaben, wie die Zahl der Beschäftigten, der Frauenanteil, die dummycodierten Branchen und der Betriebsstandort (West- oder Ostdeutschland), vor allem Angaben zur Leiharbeitnehmernutzung in das Modell ein. Im Einzelnen sind diese Variablen wie folgt operationalisiert: Die durchschnittliche Einsatzdauer der Leiharbeitnehmer wird in Monaten aufgenommen. Dichotom in das Modell eingeführt werden die Bezahlung der Leiharbeitnehmer (besser oder gleich vs. schlechter als die Stammbelegschaft), Qualifizierungsmaßnahmen bei Leiharbeitnehmern (schlechter oder teilweise schlechter vs. gleich bezogen auf die Stammbelegschaft), die Übernahme von Leiharbeitnehmern in die Stammbelegschaft, das Ersetzen von Stammbeschäftigten durch Leiharbeitnehmer, die Veränderung der Einsatzdauer von Leiharbeitnehmern (verlängert vs. gleich geblieben oder verkürzt), der saisonabhängige Einsatz von Leiharbeitnehmern, der konjunkturabhängige Einsatz von Leiharbeitnehmern und der Umstand, ob die Verleihfirma eine Tochter des Entleihunternehmens ist.

\section{ERGEBNISSE}

Das Schätzmodell zum Leiharbeitnehmeranteil erklärt ca. $24 \%$ der beobachteten Varianz und liegt damit im akzeptablen Bereich. In der bivariaten Analyse angedeutete Zusammenhänge zeigen sich auch in der multivariaten Untersuchung. Signifikante
Beiträge zur Erklärung des Anteils an Leiharbeitnehmern leisten folgende Variablen: Ein Zusammenhang besteht zwischen der Leiharbeitsquote und dem gleichzeitigen Einsatz von befristeten Beschäftigungsverhältnissen. Das Vorzeichen des Schätzkoeffizienten ist erwartungsgemäß negativ und lässt sich als Bestätigung unserer Hypothese eines substitutiven Verhältnisses beider Flexibilisierungsformen interpretieren. Je mehr Leiharbeitnehmer Betriebe einsetzen, desto weniger machen sie von befristeten Beschäftigungsverhältnissen Gebrauch. Signifikant negativ ist auch der Einfluss von Teilzeitarbeit. Haben Betriebe Teilzeitarbeit eingeführt, weisen sie einen geringeren Leiharbeitnehmeranteil auf. Dieser Zusammenhang kann sowohl mit Flexibilisierungs- als auch Kostenaspekten zu tun haben. Bei kurzfristigem Arbeitskräftebedarf bietet Teilzeitarbeit kostengünstige Möglichkeiten, das Arbeitsvolumen rasch, ohne Geldzuschläge und mit eingearbeiteten Arbeitskräften aufzustocken und kann deshalb eine Anpassungsalternative zu Leiharbeit darstellen. Teilzeitarbeit ist außerdem schlechter entlohnt als vergleichbare Vollzeitbeschäftigung (Brehmer/Seifert 2007), zudem vorrangig in Niedriglohnbereichen verbreitet, in denen ein größerer Einsatz von Leiharbeit wenig attraktiv ist.

Die Betriebsgröße und der Frauenanteil unter den Beschäftigten stehen ebenfalls mit dem Leiharbeitnehmeranteil in Zusammenhang. In Tabelle 2 ist der exakte Koeffizient der Gesamtbeschäftigten der Rundung zum Opfer gefallen. Er liegt bei $-0,002$. Nach dem Schätzmodell verringert jeder zusätzliche Beschäftigte den Leiharbeitnehmeranteil um $0,002 \%$, jede zusätzliche weibliche Beschäftigte erhöht ihn um einen ähnlich geringen Anteil. Dieser $\mathrm{Zu}$ sammenhang entsteht maßgeblich über die Konstruktion der abhängigen Variablen als Anteilsvariable. Kleine Betriebe erreichen schnell hohe Leiharbeitnehmeranteile wenn sie denn Leiharbeiter beschäftigten (Promberger 2006). Das soll nicht darüber hinwegtäuschen, dass große Betriebe bedeutend häufiger Leiharbeitnehmer beschäftigten als kleine Betriebe, was sich in

\footnotetext{
4 Huber, White oder Sandwich-Schätzer (Long/ Freese 2006, S. 86-87). Zur Absicherung wurde das Modell ebenfalls mit logarithmierten Leiharbeitnehmeranteilen geschätzt. Die Standardfehler und Signifikanzen stimmen mit dem hier vorgestellten Modell überein.
} 
dem hier vorgestellten Modell jedoch nicht beobachten lässt, da Betriebe ohne Leiharbeit ausgeschlossen sind.

Wie schon die bivariate Analyse zeigt, ist die Nutzung von Leiharbeit in den Wirtschaftsbereichen unterschiedlich ausgeprägt. Die Unterschiede bleiben multivariat bestehen, vier Wirtschaftszweige weisen einen signifikanten Koeffizienten auf. Während im Baugewerbe der Leiharbeitnehmereinsatz deutlich stärker ausgeprägt ist, spielt er in den Bereichen Handel sowie Kredit und Versicherung nur eine geringe Rolle. Dieses Ergebnis entspricht der Hypothese, dass Leiharbeit in Niedriglohnbereichen wie dem Handel eher seltener zu erwarten ist und ebenso in Betrieben mit (noch) vergleichsweise komplexen Arbeitsabläufen sowie geringer Konjunkturabhängigkeit wie im Bereich Kredit und Versicherungen.

Deutliche Erklärungsbeiträge liefert ferner die spezifische Form, in der Leiharbeit genutzt wird. Einen Einfluss auf das Ausmaß der Leiharbeit übt ihre durchschnittliche Einsatzdauer aus. Der Leiharbeitnehmeranteil steigt mit der durchschnittlichen Einsatzdauer. Noch deutlicher wird dieser Zusammenhang, wenn der durchschnittliche Leiharbeitseinsatz in den letzten zwei Jahren verlängert wurde. Diese Ergebnisse sprechen für einen Wandel in den Einsatzfunktionen von Leiharbeit hin zu längeren und nicht mehr nur kurzfristigen situativen Einsatzzeiten, wenn der Arbeitsbedarf zunimmt. In dieses Bild passt auch der Koeffizient für den saisonabhängigen Einsatz der Leiharbeit, der zur Erklärung der Leiharbeitsquote beiträgt. Die Leiharbeitsquote ist dort höher, wo der Einsatz aufgrund saisonaler Schwankungen im Arbeitsbedarf erfolgt, wie im Baubzw. Ausbaugewerbe. Diese Befunde lassen sich als Hinweise für die Hypothese verstehen, dass hohe Leiharbeitsquoten wesentlich auf die gewachsene Bedeutung der Randbelegschaftsfunktion zurückzuführen sind.

Für diese Hypothese spricht ferner der positive Zusammenhang zwischen der Höhe des Leiharbeitereinsatzes und der Substitution von Stammkräften. Wenn ferner die Übernahme von Leiharbeitern das Ausmaß dieser Beschäftigungsform positiv beeinflusst, dann deutet dieses Ergebnis auf die gewichtige Bedeutung der Erprobungsfunktion hin. Wie erwartet, wirkt ein gleicher Lohn bremsend auf den Einsatz von Leiharbeit.

\begin{tabular}{|c|c|c|}
\hline & b-Koeffizient & $\begin{array}{c}\text { Robuster } \\
\text { Standardfehler }\end{array}$ \\
\hline \multicolumn{3}{|l|}{ Allgemeine betriebliche Angaben } \\
\hline Gesamtbeschäftigte & $-0,00 * * *$ & $(0,00)$ \\
\hline Frauenanteil an Gesamtbeschäftigten & $0,03 *$ & $(0,02)$ \\
\hline Ostdeutschland & $-0,14$ & $(0,98)$ \\
\hline Gute Auftragslage & 0,63 & $(0,65)$ \\
\hline Tarifbindung vorhanden & $-0,95$ & $(0,82)$ \\
\hline Befristet Beschäftigte vorhanden & $-1,76^{* *}$ & $(0,91)$ \\
\hline Teilzeitbeschäftigte vorhanden & $-3,83 * * *$ & $(1,01)$ \\
\hline Personalabbau in den letzten zwei Jahren & $-0,81$ & $(0,64)$ \\
\hline \multicolumn{3}{|l|}{ Branchen, (Referenz: private und öffentliche Dienstleistungen) } \\
\hline Grundstoffe/Produktionsgüter & $-0,67$ & $(1,02)$ \\
\hline Investitions- und Gebrauchsgüter & 1,49 & $(1,10)$ \\
\hline Verbrauchsgüter & $-0,16$ & $(1,02)$ \\
\hline Baugewerbe & $5,65 * *$ & $(2,27)$ \\
\hline Handel (und Reparatur) & $-1,69 *$ & $(0,92)$ \\
\hline Verkehr und Nachrichten & $-0,09$ & $(1,23)$ \\
\hline Kredit und Versicherung & $-2,53 * *$ & $(1,04)$ \\
\hline Sonstige Branchen & $-2,62 * *$ & $(1,30)$ \\
\hline \multicolumn{3}{|l|}{ Angaben zum Leiharbeitnehmereinsatz } \\
\hline Durchschnittliche Einsatzdauer der Leiharbeitnehmer in Monaten & $0,29 * * *$ & $(0,06)$ \\
\hline Leiharbeitnehmer gleich oder besser bezahlt als Stammbelegschaft & $-1,66 * *$ & $(0,74)$ \\
\hline $\begin{array}{l}\text { Leiharbeitnehmer bei Qualifizierungsmaßnahmen schlechter } \\
\text { oder teilweise schlechter gestellt als Stammbelegschaft }\end{array}$ & $-0,56$ & $(0,56)$ \\
\hline $\begin{array}{l}\text { Leiharbeitnehmer in letzten zwei Jahren in Stammbelegschaft } \\
\text { übernommen }\end{array}$ & $2,02 * * *$ & $(0,65)$ \\
\hline $\begin{array}{l}\text { Leiharbeitnehmer haben in letzten zwei Jahren Teile der } \\
\text { Stammbelegschaft ersetzt }\end{array}$ & $1,63 * *$ & $(0,77)$ \\
\hline Verleihfirma ist eine Tochter des Unternehmens & 2,28 & $(1,52)$ \\
\hline $\begin{array}{l}\text { Einsatzdauer der Leiharbeitnehmer in den letzen zwei Jahren } \\
\text { verlängert }\end{array}$ & $3,48 * * *$ & $(0,91)$ \\
\hline Saisonabhängiger Einsatz von Leiharbeitnehmern & $1,49 * *$ & $(0,68)$ \\
\hline Konjunkturabhängiger Einsatz von Leiharbeitnehmern & 0,15 & $(0,68)$ \\
\hline Konstante & $7,94 * * *$ & $(2,04)$ \\
\hline $\mathrm{N}$ & & 720 \\
\hline $\mathrm{R}^{2}$ & & 0,24 \\
\hline $\begin{array}{l}{ }^{*} \mathrm{P}<0,11^{* *} \mathrm{P}<0,05 /{ }^{* * *} \mathrm{P}<0,01 . \\
\text { Quelle: WSI-Betriebsrätebefragung 2007, Berechnungen der Autoren. }\end{array}$ & & TT대 \\
\hline
\end{tabular}

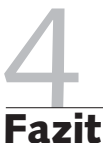

Leiharbeit hat in den letzten Jahren stark zugenommen. Diese Entwicklung hat damit zu tun, dass Leiharbeit als Flexibilisierungsvariante an Bedeutung gewinnt. Die Ergebnisse der bivariaten und der multivariaten Analyse liefern Hinweise und untermauern damit die Befunde früherer Untersuchungen (Promberger 2006), dass Betriebe Leiharbeit offensichtlich nicht mehr vorrangig nutzen, um kurzfristige Einzelbedarfe im Personaleinsatz abzudecken, sondern vermehrt auch einsetzen, um neue Arbeitskräfte zu erproben und zu rekrutieren oder um Stammbelegschaften durch flexible Randbelegschaften zu ergänzen oder teilweise zu ersetzen. Die beiden zu- letzt genannten Funktionen haben offensichtlich an Bedeutung beim Einsatz von Leiharbeit gewonnen und zur gestiegenen Nutzungsintensität von Leiharbeit beigetragen. Für diese Annahme sprechen verschiedene Hinweise. So korrelieren die Dauer des Leiharbeitereinsatzes, die Substitution von Stammkräften und auch deren Übernahme positiv mit der Quote der Leiharbeitnehmer. Auch der Befund, dass Leiharbeit in Bereichen mit höherem Lohnniveau und starker Saisonabhängigkeit (Baugewerbe) eine größere Rolle spielt als in Niedriglohnsektoren (Handel), lässt sich als Hinweis auf die gestiegene Bedeutung der flexiblen Randbelegschaftsfunktion verstehen.

Leiharbeit stellt aber auch eine Flexibilisierungsalternative zu befristeter Beschäftigung dar. Begünstigt werden diese Änderungen im Einsatz flexibler Beschäfti- 
gungsformen durch die Lohndifferenz zwischen Leiharbeitnehmern und Stammbeschäftigten, die den Leiharbeitereinsatz positiv beeinflusst. Insofern ist zu vermuten, dass eine generelle Einführung des
Referenzlohnprinzips den Einsatz von Leiharbeit begrenzen würde. Eingeebnete Lohnunterschiede dürften zumindest die Funktion der flexiblen Randbelegschaft zurückdrängen. Wie sich ein verändertes
Entlohnungsprinzip auf die Erprobungsfunktion auswirken würde, lässt sich dagegen nur schwer abschätzen.

\section{ITERATUR}

Ammermüller, A./Bookmann, B./Garloff, A./Kuckulenz, A./Spermann, A. (2003): Die ZEW-Erhebung bei Zeitarbeitsbetrieben. Dokumentation der Umfrage und Ergebnisse von Analysen, Dokumentation 03-07, Mannheim

Atkinson, J. (1984): Manpower Strategies for Flexible Firms, in: Personel Management 8, S. 28-31

Bellmann, L./Düll, H./Kühl, J./Lahner, M./Lehmann, U. (1996): Flexibilität von Betrieben in Deutschland. Ergebnisse des IAB-Betriebspanels 1993-1995, BeitrAB 200, Nürnberg

Bellmann, L./Kühl, A. (2007): Weitere Expansion der Leiharbeit? Eine Bestandsaufnahme auf Basis des IAB-Betriebspanels, Projektbericht, Berlin Böhm, W. (2005): „Zweite Belegschaften“: Mehr Flexibilität und geringere Personalkosten durch onsite management, in: Neue Zeitschrift für Arbeitsrecht 10, S. 554-564

Brehmer, W./Seifert, H. (2007): Wie prekär sind atypische Beschäftigungsverhältnisse? Eine empirische Analyse, WSI-Diskussionspapier 156, Düsseldorf

Brenke, K./Eichhorst, W. (2008): Leiharbeit breitet sich rasant aus, in: DIW-Wochenbericht 19, S. 242-252

Bundesagentur für Arbeit (BA) (2008): Arbeitsmarktpolitik in Zahlen Arbeitnehmerüberlassung - Bestand an Leiharbeitnehmern 1996 bis 2007, Nürnberg

Cappelli, P./Neumark, D. (2004): External Churning and Internal Flexibility: Evidence on the Functional Flexibility and Core-Periphery Hypotheses, in: Industrial Relations 1, S. 148-182

Delsen, L. (1996): Atypical employment: an international Perspective. Causes, Consequences and Policy, Groningen Institut für Makroökonomie und Konjunkturforschung (IMK) (2005): IMK-Report 25, Der Abschwung kommt, Düsseldorf

Håkansson, K./Isidorsson,T. (2008): A New Segment on the Labour Market. Agency workers, work organisation and health \& safety. Paper prepared for the $26^{\text {th }}$ International Labour Process Conference, 18.-20. März, Dublin

Houseman, S. (2001): Why Employers Use Flexible Staffing Arrangements: Evidence from an Establishment Survey, in: Industrial and Labour Relations Review 1, S. 149-170

Jahn, E./Rudolph, H. (2002): Auch für Arbeitslose ein Weg mit Perspektive, in: IAB-Kurzbericht 20, Nürnberg
Kalleberg, A. L. (2001): Organizing Flexibility: The Flexible Firm in a New Century, in: British Journal of Industrial Relations 4, S. 479-504

Keller, B./Seifert, H. (2007): Atypische Beschäftigungsverhältnisse. Flexibilität, soziale Sicherheit und Prekarität, in: Dies. (Hrsg.): Atypische Beschäftigung - Flexibilisierung und soziale Risiken, Berlin, S. 11-26 Klemm, M./Sandig, C./Schuberth, J. (2008): Intensivnutzung von Leiharbeit in der BRD: Fallstudien zu Motivlagen und Folgen der Intensivnutzung der Arbeitnehmerüberlassung in Betrieben des verarbeitenden Gewerbes (mit Schwerpunkt Metall- und Elektroindustrie), Erlangen Kvasnicka, M./Werwatz, A. (2003): Lohneffekte der Zeitarbeit, in: Bundesarbeitsblatt, S. 2-10

Long, S./Freese, J. (2006): Regression Models for Categorical Dependent Variables using Stata, Texas/USA

Massa-Wirth, H. (2007): Zugeständnisse für Arbeitsplätze? Konzessionäre Beschäftigungsvereinbarungen im Vergleich Deutschland - USA, Berlin Miegel, M./Wahl, S./Schulte, M. (2007): Die Rolle der Zeitarbeit in einem sich ändernden Arbeitsmarkt, mimeo, Bonn

Nienhüser, W./Matiaske, W. (2003): Der "Gleichheitsgrundsatz" bei Leiharbeit - Entlohnung und Arbeitsbedingungen im europäischen Vergleich, in: WSI-Mitteilungen 8, S. 466-473

Promberger, M. (2005): Leiharbeit. Flexibilitäts- und Unsicherheitspotenziale in der betrieblichen Praxis, in: Kronauer, M./Linne, G. (Hrsg.): Flexicurity. Die Suche nach Sicherheit in der Flexibilität, Berlin, S. 181-204 Promberger, M. (2006): Leiharbeit im Betrieb. Strukturen, Kontexte und Handhabung einer atypischen Beschäftigungsform, Nürnberg,

http://www.boeckler.de/show_project_fofoe.html?projectfile= S-2002-418-3.xml\#veroeffentlichungen

Promberger, M. (2007): Leiharbeit. Flexibilität und Prekarität in der betrieblichen Praxis, in: Keller, B./Seifert, H. (Hrsg.): Atypische Beschäftigung - Flexibilisierung und soziale Risiken, Berlin, S. 127-144 Seifert, H. (1988): Zeit- und Leiharbeit aus gewerkschaftlicher Perspektive, in: Deutsches Institut Zeitarbeit (Hrsg.): Zeitarbeit und überbetriebliche Beschäftigung als Element umfassenden sozialen und wirtschaftlichen Wandels, Bonn, S. 131-148

Seifert, H./Massa-Wirth, H. (2005): Pacts for Employment and Competitiveness in Germany, in: Industrial Relations Journal 3, S. 217-240 Urban-Crell, S./Schulz, C. (2003): Arbeitnehmerüberlassung und Arbeitsvermittlung, München 